UDC 004.8

\title{
Challenging the Uniqueness of Being by Cognizing
}

\author{
Edward M. Pogossian \\ Institute for Informatics and Automation Problems of NAS RA \\ e-mail: epogossi@aua.am
}

\begin{abstract}
Humans become powered enough to question the further types of their being while there are no ways to resolve the mystery of being of cellular realities (cellulars) predetermined by a type of programs, genomes, and their universal processors.

Acknowledging that genomic reproduction can not be originated by a chance, the kernel of effective cognition is universal for being in the universe and mental models can be reduced to basic classifiers, in what follows, we continue to challenge the uniqueness of human cognizing arguing possibility of origination of basic classifiers in frame of fundamentals of physicists followed by constructive formation of mental systems composed from those basic classifiers.

Keywords: Cellular, Classifiers, Relationships, Cognition, Constructive, Modeling, Mentals, Neuron nets.
\end{abstract}

\section{Introduction}

\subsection{Questioning cognizing}

1.1.1.Cognizing, in general, are mental doings on revealing, accumulating and processing models of realities aimed to support certain beings while cognizers (cogs) are, we assume, are a type of mental systems (mss) represented by those mental doings [1,2].

At present, the highest human cogs (hCogs) approach to constructing adequate models of cells and genomic reproduction of cells.

Simultaneously, the models of hCogs become capable of transitioning to the higher mental doings D" for a variety of given starting doings D'.

1.1.2. Thus, we approach to questioning the power of hCogs as follows:

-Can maxD" be equal to doings of hCogs? Particularly, can maxD" be equal to doings of hCogs in constructive modeling of cells and their incremental development?

- What doings have to be necessarily included in minD'?

- Can minD' be equal to doings of certain min classifiers ( $\mathrm{minCl})$ ? 
-Can minCl be originated in frame of acknowledged by physicists fundamentals of universe?

1.1.3. Assuming that mss can be composed from certain minCl and maxD" can be equal to doings of hCogs it follows that

-maxD" include doings allowing to construct models of transition from D' to D", or models of formation of mental doings D" (mfrms D") given D' (due to the assumptions on equality of maxD" and doings of hCogs and a constructive ability of hCogs to transit from D' to D")

-mfrms can be incrementally composed from minCl (due to the reasoning in $[2,4]$ that all mss (including mss of formation of mss) are reducible to elementary classifiers minCl).

In other words, it was assumed that non-cellular realities are possible that given certain elementary classifiers can construct mss comparable by complexity with the highest ones of humans.

1.1.4. Adding to the above ones a new assumption that minCl can be originated from the fundamentals of matter and energy it can be assumed that in frame of certain realities of the early universe a chain of mss rooted in minCl can be constructively formed up to the highest hCogs.

And it is not excluded that those realities are ones referred in [3] as lemuroids.

And it would not be excluded that those lemuroids found perspective to create new additional to them carriers of the roots of their being as, at present, they are known for the cellulars.

Particularly, they could create and implement genomic programs and procedures of diversified reproduction of cells.

1.1.5. Concisely, the hypothesis on the highest constructive cogs (hcCogs) states that noncellular realities, say lemuroids, with the highest hCogs can be originated in frame of basics of physicists and those

- lemuroids can diversify the stability of their being creating the cellular carriers of their roots with the abilities of preserving those roots as, at present, we observe them for the cellulars.

The hypothesis hcCogs, if right, will provide a constructive way to avoid the mystery of origin of cellulars by a chance.

1.1.6. Positive premises for the hypothesis are inspired by advances in mental modeling and by attempts to reveal the most necessary requirements for early cognizing $[1,2,4,5]$.

Another premises follow the conviction of Piaget [6] on the universality of procedures and means of development of humans, the hypothesis in [7] on plurality of lines of evolution stating that in parallel with evolution of cellulars there are evidences on the unique line of evolution of viruses as well as the beliefs of Buddhist in preceding us highly advanced lemuroids [3].

It is also worth recalling that the hypothesis is consistent with an irresistible conviction of the vast majority of theories and religious on existence of the creators of cellulars.

\subsection{Constructive modeling of formation of classifiers.}

1.2.1. So far, sub problems of hcCogs could be identified as ones of constructive modeling of

- first, the origination of $\mathrm{minCl}$ in early universe, and

- second, the formation of chains of incrementally complicated mental doings rooted in minCl and comprising ones of hCogs.

1.2.2. Solving the first problem we assume that minCl are ones formed over identified outputs of innate classifiers, or imprints, particularly identified sensors, and identified classifiers formed in life times and complex mss can be the compositions of those minCl [2].

Therefore, at the next step a chain from the fundamentals of physicists to minCl have to be provided. As the part of that chain in [8] we argue that in early universe certain durable in time realities are possible that can accumulate imprints of other realities and be able to preserve that ability gaining energy from others, i.e., be not entropic, or negentropic, by [8]. 
Thus, the next target on the way to minCl have to be ability to classify those accumulated imprints and a premise to it can be findings in [5] that elementary units of information, in our interpretation elementary classifiers, can be originated in the most early universe.

1.2.3. In what follows we address the second problem and look for models for the formation of complex mss rooted in minCl acknowledging that mss are gained

- inherently and by learning in life times, i.e., by revealing, discovering and acquiring of new classifiers and enhancing the quality of classifiers while

- revealing of new mss is mainly inductive, therefore highly personalized and approximate.

At first, we interpret introduced in $[1,2,4]$ constructive models of mss, mentals, by the basics of attributive classification of theoretical computer science linking to each other, particularly, the attributes and the types of classifiers of mentals, the teaching samples of experts and the matrices of stored prints, addressing also to the ways of regular extension of attributes and to the origin of non- expert dependent criteria to split realities as positive or not.

Then, referring to some relevant models of classifying we provide an approach to inductive formation of two place relationships that being the basic units of mentals, analogously with ones in clauses of languages, induce guides of formation of mentals, particularly, in the artificial neuron nets (ANN) modes.

Finally, we discuss the formation and acquisition of systemic classifiers and the ways of coping with imperfect classifying.

\section{Refining Models of Mental Classifying}

2.1. Let recall some basics of models of mental classifying introduced in $[1,2,4]$ followed by their refinement.

2.2.1. At first, let recall that doers are do-classifiers $\mathrm{Cl}$ if indoms are split into two classes $+\mathrm{Cl}$ and ?Cl; otherwise they are corresponders, cors.

Apparently, identifiers of do-classifiers $C l$ by themselves are sufficient to indicate their classes of equality, i.e., the positives $+\mathrm{Cl}$, while classes of cors can be indicated by pairing those identifiers with the corresponding outputs.

Classifiers of n-tuples of nominals are $n$-place relationships, or nrels.

Since later we mainly address to classifiers of nominals they will be interpreted as 1rels while 2rels will be shortly named rels.

Rels $(a, b)$ can or cannot depend on the orders of their arguments.

2.2.2. Then, recall that nominated wrt controllers Cns outputs of doers, particularly, sensors, controllers or effectors, are named otids while sets of otids of doers d are the alphabets of $d$.

And sets of otids comprised from only some representatives of alphabets $A 1, A 2, \ldots, A n$ of doers $d 1, d 2, \ldots, d n$ are words in $A 1, A 2, \ldots, A n$.

2.2.3. Let ces be sets of controllers Cns, effectors Efs and sensors Sns nominated wrt to Cns.

Then doers $D$ over IDs of ces, or cesdoins $D$, are doers $D$ nominated wrt to Cns while indoms of $D$, Efs, Cns are words in alphabets of the outputs of $D$, Cns, Sns.

2.2.4. Bundles of otids of Sns, Cns and cesdoins at time $\mathrm{t}$ are $t$ prints comprised into certain stores Pns and nominated wrt to Cns.

2.3. So far, in $[1,2,4]$ classifiers were introduced of the types of :

-sensors xSns,

-genomic goals $\mathrm{xGn}$, including evolutionary approved goals, or classifiers of utilities wrt the roots and chains of consequent classifiers, goals, wrt preceding ones,

-genomic classifiers $x$ Clgn uniting Sns and sGn, 
-goals $G l$ corresponding to classifiers $x C l t$ learned in the life time (1t) of $x$ and uniting do classifiers $x d o C l$ by themselves and systemic classifiers $\mathrm{xsCl}$ induced by ad hoc $d o$ classifiers of the constituents of mentals of the thesauruses $x T h$, -goals $x G$ uniting $x G n$ and goals $G l$ and, finally, -attributes $x A t r=x(C \operatorname{lgn} U C l t)=x(G n U G l)$ and comprising all do classifiers of $x$ available at time $t$.

Note, that genomic goals are classifying utilities wrt the roots but do not include classifiers of roots themselves which are ones of high layers of humans studying the roots.

2.3.1. The unit classifiers $\mathrm{Cl}$ of $x$ Atr, the attributes, at time $t$ output their IDs for the input positives of $+C l$ at $t$, i.e., when they are activated by positives of $+C l$ at $t$.

Bundles of IDs of activated at $t+\Delta$ attributes, or $t$ prints, of $x$ are accumulated at stores $x S P$ since the moment $t=0$, i.e., the time when $x$, we assume, can be classified as organisms, while $\Delta$ is the discretion time sufficient for outputting of attributes of any layers depended from activation of attributes of the lower layers.

The diversity of all possible prints for $x$ Atr at $t$ comprise the space $\mathrm{xSP}^{*}$ of possible prints at $t$. Apparently, $\mathrm{xSP} *$ is a representation of the universe $\mathrm{xU}$ of $x$ at $t$.

2.4. Communities $C$, we assume, have an ability to address and to certain extent control the classifiers of the members of $C$.

Thus, we can assume, that $C$ can control the unions of classifiers of the types of ones of the members of $C$, i.e., $C$ can control classifiers of the types of $S n s$ and $G n$, since the members of $C$ have almost identical genomes, then the types of doClC, sClC, ThC, GC, GlC and AtrC .

We assume also the common for $C$ universe UC and space of prints SP* uniting $\mathrm{xU}$ and xSP* of all $x @ C$, correspondingly, and determined by AtrC.

2.4.1. Mental doings of members $x$ of $C$ these days can be analyzed in depth mainly personally while analyzing the integrative power of $C$ it is worth addressing to the unions of mental doings of x@ $C$.

For convenience, therefore, let's address to the ideal members $z$ of $C$ identified by classifiers of Sns, Gn, Th, Clt, G, Gl, Atr, SP* and U coinciding with analogous integrative ones of $C$.

2.5. While prints and their spaces are determined by Attributes at $t$, the outputs of sensors, the percepts, united with the outputs of genomic goals Gn and the outputs of classifiers Clt, i.e., the learned prints, determine the space of genomic prints SPgn and the learned space of prints SPl, correspondingly, with the powers $2^{n}$ and $2^{m}$, where $\mathrm{n}$ and $\mathrm{m}$ are the powers of SnsUGn and Clt at time $t$, correspondingly.

Apparently, the spaces of prints SP at $t$ will have the power $2^{n+m}$.

\section{Referring to Attributive Classifying}

3.1. Mental learning to classifying includes reveling, discovery, in general, formation of classifiers by members $x$ of communities $C$ in the life times, where learning, particularly the inductive one, necessarily assumes a unique contributions of $x$ to that formation, and includes acquisition of ready to use classifiers of $C$ with more standardized while minimized doings of $x$.

3.1.1. Addressing to learning of classifiers (LC) $\mathrm{Cl}$ of $z @ C$ it is worth, first of all, recalling classifiers in advanced models and theories where classifiers are represented by compositions of certain attributes for the followed then transition of the statements of those theories to the modeling LC by mentals.

3.2. Let's also remind that mentals aim to represent, at least, realities having corresponded and identifying them units of languages (ideally, of the natural ones) and represent consistently with the scope of those units. 
For example, mentals representing chess positions have to include relevant classifiers of game trees, the best strategies or their search algorithms.

3.2.1. In logics classifiers can be interpreted as prepositional formulas and LC corresponds to the logical inference of theorems from the given axioms.

The axioms can be interpreted as classifiers with certain trustful utilities while theorems as ones whose utilities have to be inferred from axioms applying trustful logical rules, for example, modus pones.

Apparently, the utilities of theorems will be transferred from ones of axioms, thus, compared with the axioms will not enhance their reliability wrt to, for example, genomic goals.

3.2.2. Instead of logical rules the inference of new classifiers from ones with certain utilities can proceed by means of some case effect regularities.

For example, from the given chess positions $P$ strategies can infer $P$ from positions $P^{*}$ with already known, thus, transferring those utilities to $P$ as well [9].

3.2.3. Along with targeting inferences of the theorems or winning positions the targets can be the classifiers of inferences themselves.

For example, in chess game trees for the given positions $P$ the classifiers of proper in $P$ strategies can be questioned $[9,10]$.

Search of classifiers of proper strategies in the entire thesauruses of mentals, seems, can be analogously formulated.

3.2.4. Some studies by N.Nepeivoda question the transition from the classifiers of inferences to the classifiers of algorithms of those inferences.

Another ones focus on the transition from classifiers given implicitly to the explicit ones.

For example, in [11] the implicit classifiers can be given by sets of equations and the explicit ones are searched using the theorem of the fixed point among the space of codes of all possibly relevant classifiers.

3.3. A type of representations of prepositional formulae (in fact, the classifiers) are Boolean functions [12].

Boolean models of FC address, particularly, to inductive restoration of Boolean functions $\mathrm{Cl}$, potentially being justified, say, by experts or oracles, by consistent and representing $\mathrm{Cl}$ matrices of samples of Boolean vectors $\mathrm{mv}$ either from $+\mathrm{Cl}$ or not.

Algorithms of generalization of matrices $m v$, inductors, and algorithms of inductive inference of $\mathrm{Cl}$ by regular processing of inductors over the sequences of matrices $m v$ and the frontiers of approximation of target $\mathrm{Cl}$ are widely analyzed and represented, particularly in [9].

For example, a type of inductors is compacting $m v$ matrices representing them by equal prepositional formulae $\mathrm{Clgi}$ of the types of conjunctive of disjunctive forms composed by logical operators from the attributes.

Let emphasize that the matrices $m v$ provided to the inductors of prepositional formulae (IPF), assumingly, have to be enough representable to restore those formulae, can be available entirely at ones, part by part, repeatedly or not, etc., following some heuristics of IPF.

3.4. Learning in ANN is by inductors and inductive inferences of certain types as well [9].

Addressing to learning by back propaganda (LBP) it can be classified as a type of inductive inference where the outputs of target classifiers built by certain inductors is cyclically compared with ones expected by the teachers and corresponding to coincidence or not of the outputs with the expectations the cycle of applying the inductors is continued or not.

A uniqueness of used now LBP compared with IPF is in the sequential, one by one provision of the vectors of the matrices $m v$.

It could be an advantage of LBP or analogous inductors when the analysis of the entire matrices meets some difficulties. 
3.5. Assuming that LC in addition meets certain statistical requirements, the chains of consequent statements on the formation and quality of classifiers are presented, particularly in [13].

\section{Continuing Refinement of Models of Mental Classifying}

4.1. Mentals are defined as systems over the outputs of growing up attributes where the basic ones comprise genomic classifiers including sensors and ones formed evolutionary.

Assumingly, genomic classifiers are the permanent, lasting constituents of the attributes while new classifiers enriching them not necessary can be involved into ongoing classifying.

4.1.1. Sensors have a unique physical nature related to vision, touching, hearing, others.

Interpreting human vision, for example, visual sensors are a type of neurons linked by weighted synapses to the light sensitive units of retina analogously with pixels.

The distribution of synapses over retina and their number for each of those primary neurons, sensors, seemingly, have to be the normal one to ensure equal inputs to the sensors from all parts of the retina while their density, analogously with eyes, have to be much higher for the neurons linked to the center of the retina than for the periphery ones.

It is not excluded that the weighs of synapses of sensors can change to meet regular peculiarities of the inputs analogously, for example, with the facets of frogs specialized to react only to the particular inputs of types of lines, motions, others.

4.1.2. For further references the above assumptions we comprise as follows

Ass1. Genomic classifiers are permanently involved in new classifying while ones of the type of sensors are specialized in representation of certain fields of realities and vary in the types of representations of each field.

4.2. In machine learning of classifiers $\mathrm{Cl}$ positives of $+\mathrm{Cl}$ are provided by experts while in autonomous inductive learning the sources preliminary have to be refined .

The given sources of positivity learning can be processed on the base of the positives provided either by matrices of samples of $+\mathrm{Cl}$ similar to the case of Boolean IPF or sequentially similar to LBP in ANN.

4.2.1. In refining positivity of realities wrt classifiers we believe they comprise positives $+C l i$ if they have common relationships with certain identified utilities of humans that can be interpreted as goals.

For example, positives of the class Sweets necessarily have to provide utilities being sweet.

Those utilities can address to the tangible realities of the classes Sweets, Soars, Cold, Hot, etc. or to the classes Nutrients, Damagers, etc. but they can also refer to not tangible, highly abstract goals like Classify All Over.

In the early stages of childhood those classifiers are senso-motoric by Piaget and are tidily governed by the right hemisphere. And only at later stages they incrementally enhance their abstractness and can be located in the left hemisphere as well.

4.2.2. Recall also that in attributive formation of classifiers $C l$ there are certain criteria, goals gi, wrt which those $\mathrm{Cl}$ are formed.

For example, inductors in Boolean models generalize matrices of Boolean vectors mv consistent with positives $+\mathrm{Cl}$ of target classifiers $\mathrm{Cl}$. In those models the question of positivity or not of vectors of $m v$ is prearranged by humans and their origin is not discussed.

4.2.3.The above notes we resume by the following

Ass2. Formation of do classifiers Cl of $\boldsymbol{z} @ C$ at time $t$ is always processed wrt certain goals $g$ available to $z$ at $t$.

CIr.1.2. Do classifiers $\mathrm{Cl}$ of thesauruses cisTh are always accompanied by certain explicit or implicit goals $g$ representing ones guiding the formation of $\mathrm{Cl}$. 
4.2.4. Thus, in modeling or processing of $d o$ classifiers $C l$ it is inevitable to be not aware of the goals $g$ guiding their formation.

And it is reasonable to assign Clgi to classifiers formed wrt goals gi while, analogously, mpgi, or matrix of prints wrt gi, to assign to the sub stores of the sets of $t$ prints SP comprising ones accompanied by activation at $t$, i.e., with taken place, goals gi.

4.2.5. Addressing to inductors the above let induce the following corollary

Clr.2.2..Inductors have to be interpreted as algorithms providing hypothesis on classifiers Clgi by extension of given matrices mpgi assumingly consistent with + Clgi [9].

4.2.6. Questioning goals accompanying the systemic classifiers $\mathrm{sCl}$, analogously with the $d o$ ones, let's recall that they are induced by mss $m$ (or modeling $m$ mentals $m$ ') which, in turn, are composed of mdoers ( mdoins) why the goals of $m\left(\mathrm{~m}^{\prime}\right)$ and $\mathrm{sCl}$ is reasonable to determine in certain dependency from the goals of those mdoers (doins).

4.3. Recalling the views "Knowledge is the Strength", "Seeing by the Mind [14]" we interpret them as "Seeing by the Classifiers", thus, following the assumption.

Ass3. All ad hoc classifiers can have a positive impact on the formation of new classifiers

4.3.1. Particularly, it follows that in mpgi based IPF and in sequential LBP formation of new classifiers it is recommended to take into account attributes corresponding to the representation of all as ad hoc classifiers.

Apparently, what parts, in general, of lengthy prints have to be involved in the formation of classifiers and how to do that need special refinements.

For example, Boolean inductors before compacting mgi matrices into equal conjunctive of disjunctive forms compress the attributes of initially given mpgi to the min necessary, i.e., min tests [12], to preserve the correctness of mpgi.

\section{Formation of Relationships}

5.1. The fundamental constituents of mentals are relationships and doins that, in turn, can, in principle, to be reduced to $n$-place do classifiers, relationships, over the nominals $[1,2,4]$.

While the formation of 1-place classifiers is widely illuminated it needs to be refined, at least, for 2-place ones as it follows.

5.2.1. Mpgi based IPF can form 1-place classifiers Clgi analyzing only positives of mpgi but become expectably more consistent with Clgi if accompanied by matrices -mpgi representing not positives, negatives of $\mathrm{Clgi}$, i.e., realities not activating goals gi.

5.2.2. Rels, in general, classify being in space and time of two classes of realities represented by their IDs.

So, Arels 1B means that nominals with IDs A relate to ones with IDs B as rels1.

Analogously with IPF formation of 1-place classifiers by matrices $\pm m p g i$ the IPF formation of Arels1B can be proceeded for mpAB matrices of prints with positive values of attributes $A$ and $B$ split into two parts \pm mpAB, where the prints of the $+m p A B$ parts meet the rels1 while the rest of mpAB, i.e., -mpAB don't.

Apparently, classifiers $\mathrm{Cl}$ correctly classifying $\pm \mathrm{mpAB}$ are algorithmic while the problem of classifiers $\mathrm{Cl}$ consistent not only with \pm mpab but with the target Arels1B does not have universal algorithmic solutions and for each ArelsB has to be uniquely analyzed given additional constraints.

5.3. Classifiers Arels1B identify rels1 between the particular classes of realities with IDs A and B.

For $\pm m p$ based formation of classifiers of rels1 as themselves, in general, i.e., formation of $x$ rels $1 \mathrm{y}$ where $x$ and $y$ can be arbitrary IDs, the +mprell matrices can comprise available prints 
of SP stating the presence of activated attributes A rell B between any IDs A and B while mprell have to include certain opposite prints where, at least, there is no rels 1 between those IDs.

5.4. LBP formation of $A$ rels1B and $\boldsymbol{x}$ rels1 $\boldsymbol{y}$ can be proceed analogously with the sequential provision and learning to the prints of $\pm m p$ matrices.

Particularly, given at $t$ realities $r$ to the interface of, say RGT Solvers [17], form in the discretion time $\Delta$ prints caused by $\mathrm{r}$ that are comprised from IDs of all activated at $t$ classifiers, and thus, the attributes.

Then, LBP have to be processed for each of those realities $r$ at the interface, causing, in fact, certain mpgi matrices of prints, until target classifiers Clgi will be formed.

And those new formed Clgi enrich the attributes to be used in further LBP.

5.5. Let's state the above findings in formation of rels as follows:

Ass1. Inductive algorithms of formation of rels can be particularly constructed of the types IPF or LBP and based on mpgi matrices, that are not necessary numerical and can be both, between personalized, classifiers of unique realities and between variables of classifiers, thus, representing rels themselves,

Ass. 2. The above algorithms can be processed, particularly, in the frame of ANN

Ass. 3. The analogies in formation and storage of rels in NN can essentially enrich the ones for mentals and ANN while those models can enrich understanding of $N N$.

\section{Learning Mental Systems. Formation}

6.1. Systemic classifying is based on a variety of doings by the constituents of mentals, i.e., doins, including a variety of types of do classifiers .

Thus, the analysis of learning to mentals necessarily enlightens learning to the constituent classifiers of mentals as well as learning to systemic classifiers induced by the mentals.

6.1.1. In formation of mentals let us accept that the formation of new mentals based on already given ad hoc ones and formation of the terminals of mentals, the doins [1,2,4], not coincide why in what follows they are discussed one by one.

6.2. For formation based on the ad hoc mentals let's recall that mentals extend abstract classes and their storages why the thesauruses cesTh are analogous to the libraries in OOP, say, in Java.

So, it is worth reminding what the units of cesTh are and how they are assumingly stored to transfer their frames to the formation of mentals.

6.2.1. Namely, in $[1,2,4]$ it is assumed that the units of cesTh represented as the nodes A of cesnets, are stored with IDs of A, the classifiers of IDs of A, IDs of rels of A with other nodes B along with IDs of those B.

Nodes A corresponding to ces abstracts $d$, in addition, contain either the decision makers of $\mathrm{d}$ or the references to them.

And apparently, nodes corresponding to ces abstracts of cesnets or in cesTh will coincide with abstract classes of Java in the case when their rels with other nodes of cesnets are restricted by "attributed", "parented" and "done by" ones.

6.2.2. The analogy between storages of mentals and OOP libraries let us assume as follows:

Ass: For the given ad hoc mentals the new ones can be formed analogously with abstract classes but in contrast with them using only standard OOP rels: attributing, being and doing, mentals can be formed using the entire range of constructively modeled rels of natural languages.

Ideally, those have to include all rels with IDs represented by the units of natural languages. 6.2.3. Note, that in mpgi based formation of classifiers $C l g i$ the attributes also represent already available mentals while the formulas formed, say by IPF, are, in fact, systems comprised from those attributes and logical rels between them. 
6.2.4. Note, also that another option in formation of new mentals for the given ad hoc mentals can be the abstractions of those mentals outlined in $[1,2,4]$.

Namely, $j$-th abstractions and total abstractions of a mentals $G$ are defined in the way that the 1 st abstractions of a mentals $G$ with nodes a at some layer $k$ of ces nets Nts could be $a 1, a 2, \ldots$, an rooted mentals $G 1, G 2, \ldots, G n$ of all subsystems of $G$ with $a 1, a 2, \ldots, a n$ at the $k+1$ layers of Nts and connected to $a$, etc.

6.3. In formation of mentals not reducible to others, let's remind that they comprise the terminal nodes of decompositions of the given mentals as follows.

6.3.1. Decompositions of 1 st depth, or 1 st decompositions, of a mentals $G$ having nodes $a$ at some layer $k$ of cesNts are $a 1, a 2, \ldots$, an rooted mentals of all subsystems $G 1, G 2, \ldots, G n$ of $G$ with nodes $a 1, a 2, \ldots$, an of $k-1$ layers of Nts connected to $a$.

And if $G 1, \ldots, G n$ are $i$-th decomposition of $G$ then $i$-1-th decomposition of $G$ will be the union of 1 st decompositions of $G 1, \ldots, G n$.

Apparently, the terminal decompositions of $G$ will be comprised from ces bnominals of Nts. And the unions of $i$-th decompositions of $G$ for $i=1, . ., k-1$ comprise total decompositions of $\mathrm{G}$.

Let's recall also that ces bnominals are deifined as follows $[1,2,4]$.

For the given controllers Cns, effectors Efs and sensors Sns nominated wrt to Cns, or ces, doers $D$ over IDs of ces, or cesdoins $D$, are doers $D$ nominated wrt to Cns while indoms of $D$, Efs, Cns are words in alphabets of the outputs of D, Cns, Sns.

Basic ces nominals, or ces bnominals, include cesdoins $D$ united with Cns, Efs,Sns,Pns nominated wrt Cns.

6.3.2. Thus, the terminals of mentals are doins $[1,2,4]$ that can be either of 1 or 2 place rels, or elementary corresponders, regs, or the compositions of regs and rels, the abstracts.

Since the formation of 1-2 place rels/classifiers was refined in above, let's now address to the formation of regs and abstracts.

6.4. First, let us remind that abstracts are reducible to regs and rels between them $[1,2,4]$.

Indeed, systems of ces bnominals, or scesbns, are systems over Nls, Rls where Nls are cesbns and the totalities of scesbns comprise cesnets Nts.

Then, the following types of scesbns can equally correspond to algorithms, say, in Markov or other equal modes.

Equal to rules by Markov are types of doins, regularities, or regs, corresponding certain otids to only some selected words of the indoms.

Algorithms are scesbns comprised from regs by rels similar to ones comprising rules into algorithms by Markov [15,16].

Scesbns algorithms, in fact, deepen the definition of ones by Markov detailing the origin of the rules. Namely, if Markov algorithms are defined starting from the given basic alphabets the scesbns ones assume certain sensors providing those basic alphabets.

Scesbns of the types of "abstract classes", referring, say, to ones in Java, are systems of algorithms, or methods, in rels of the types: "attributed", "parented" and "done by", with other abstract classes.

Abstracts expand abstract classes by allowing arbitrary rels of Rls with other abstracts.

Finally, packages of abstracts and their libraries are mimicking the ones in Java.

6.4.1. From the above it follows that the final stages of formation of terminals of mentals are reduced to formation of regs .

Some of those regs can be interpreted as space, static rels what, in general, are known in logics, stating that functions can be interpreted as predicates and vice versa.

Recall also that methods of the abstracts, if represented by Markov algorithms, are the systems of not static rules, regs of the types of "realities $y$ follow realities $x$ ", " $y$ is function of $x$ ", 
" $x$ cause $y "$ ", etc., where their appearance in time is essential and cannot be reduced to the ones in space.

Nevertheless, those regs can be reduced to a type of time, case-effect, dependency rels as it was argued in $[1,2,4]$.

6.5.Uniting the above analysis with ones in [2] it can be stated that

Ass1. Mentals can be represented as systems of do classifiers of the types of one, two place rels.

Clr1.1. Formation of mentals can be reduced to a formation of do classifiers of the types of one, two place rels.

Clr2.1. The storages of thesauruses cesTh of mentals induced by the libraries of OO abstract classes can, in principle, be organized as storages for representing those mentals systems of do classifiers of the types of one and two place rels.

6.5.1. Since the above assumption does not refer to a particular representation of 1-/2-place resls they can, particularly, be realized by a certain module ANN, or a unit ANN (unn), thus, allowing to state

Ass2. Mentals, in principle, can be adequately modeled by ANN that, at first, represent 1-/2place rels of mentals equal to unit ANN, then, compose those units into ANN equal to the basic constituents of mentals and, finally, unite those constituents into ANN equal to the target mentals.

To make the above reduction not only existing, in principle but also constructive, the storage and linkage of the unit ANN into the target compound ANN have to be refined.

6.5.2. The linkages of mentals are based on the recalling of IDs of mentals, similar to OOP,

In general, a way for assuring the linkages in ANN could be the modeling of ones in OOP.

Another more realistic approach can be based on modeling of linkages in $\mathrm{NN}$ where the rels between neurons of the same or different layers, assumingly, are represented by synapses while the amount of those synaptic links of $\mathrm{NN}$ of any human concurs with the amount of particles in the universe.

To model rels of NN based on synapses recall our above approach to formation of rels where, at first, rels Arels $1 B$ are formed between personalized, classifiers A and B of unique realities, then, using those ground rels are formed rels $x$ rels $1 y$ between the variables $x, y$, of any classifiers to represent the rels themselves.

The above allows to assume that rels in NN can be represented as follows

Ass3. Given $A$ and $B N N$ classifiers the relsl between $A$ and $B$ can be formed as a new $N N$ classifier $A$ rels1B linked by synapses, necessarily, to $N N$ classifiers of $A, B$ and, expectedly, to other ones.

This assumption, assumingly, can be a hint to the models of mental classifying, either of the types of mentals or ANN, that like to children, will incrementally enhance the complexity of learned rels starting with the units of the types of conditional reflexes, then, step by step uniting them into more and more compound classifiers.

\section{Learning Mental Systems. Acquisition}

7.1. Outlining the second dimension of mental learning, the acquisition of mentals, let remind, that while inductors are important for communities $C$ in revealing, discovering new classifiers the majority of classifiers are transferred from generations to generations of their members hand to hand assuming the abilities to explain, teach, tutor for the knowledgeable members of $C$, the experts, while for the students the abilities, at least, to acquire those classifiers represented mainly by the text in their languages.

7.2. Thus, at least, two types of acquisition of classifiers $C l$ of $C$ have to be analyzed

- hand to hand when $\mathrm{Cl}$ is only a personal expertise of members of $C$, and 
- estranged, when $\mathrm{Cl}$ are estranged from members of $C$ by certain records and can be learned by others if certain keys of $\mathrm{C}$ were preliminarily hand to hand acquired.

Those records can be thesauruses represented by the texts in languages while the keys can comprise the alphabets and basics of those languages.

7.3. Constructive models of estranged acquisition of human classifiers present translators, interpreters from $\mathrm{OO}$ programs allowing the classifiers $\mathrm{Cl}$ of abstract classes represented, for example in Java, to correspond equal to $\mathrm{Cl}$ classifiers in computer codes.

7.3.1. Recalling that mentals extend abstract classes, the OO models of estranged acquisition of OO classifiers can be extended to analogous ones to acquire classifiers of mentals which wrt $\mathrm{OO}$ programs extend rels attributing, parenting and doing to all those that can be formed, particularly inductively, pursuing to cover as much rels of natural languages as can be modeled.

7.3.2. Note, that the models of natural languages (NL) like UNL, while analogous to mentals, tend to extend $\mathrm{OO}$ rels and represent all rels of NL operate, in fact, only with the IDs of mental classifiers of humans represented in NL, thus, are, at least, restricted in adequate modeling of mental doings [2].

7.4. The stages of estranged acquisition of human thesauruses presented, ideally by the encyclopedic texts of Wikipedia, in our vision, apparently, have to include transformation of those texts into isomorphic composition of clauses and transition from the texts to equally represented them mentals

A variety of heuristics will inevitably be involved in those acquisitions, so the question arises of scaling of quality of acquisition for the proper choices.

An approach to do that choice in frame of RGT Solvers could be as follows.

7.4.1. RGT iSolvers personalized by their acquisition algorithms iAlgs learn Wiki and form thesauruses iTzs.

An approach to choose the best *Solvers can be arranged by Tournaments between iSolvers, particularly in ways analogous to ones in [9], namely:

-the diversity of all possible learning menatls iAlgs can be enumerated

- by tournaments analogous to [9] an exhaustive wrt all sequences of proper bundles of iSolvers can be arranged to compete for the best RGT *Solvers wrt to a comprehensive diversity of RGT problems

-as it is proven in [9] those competitions will converge to the best RGT *Solvers.

7.4.2. As a step of the above project, the idea can be examined preliminary for *Solvers/chess where Wiki will be reduced to the Chess Repository of [19] expanded by chess books (CR+).

Based on the experience of acquiring chess classifiers [18] iSolvers/chess learning then competing by the [9] tournaments are guaranteed to converge to the best in the class *Solvers/chess.

\section{Questioning Perfectness of Classifying}

8. 1. At first, recall the assumption that classifiers are always accompanied by certain explicit or implicit goals representing ones guiding their formation why in modeling or processing of classifiers it is inevitable not to be aware of those goals.

Then, all the goals are "rooted", i.e., the roots induce a variety of classifiers to govern the doings including the genomic goals $G n$ classifying evolutionary approved utilities wrt the roots and classifiers/goals $G l$ learned in the life time.

8.1.1. Ideally, it could be assumed that all classifiers were formed in the genomic spaces of humans and wrt the genomic goals $G n$ that are highly identical for all of humans.

In other words, we assume that perfect classifiers wrt gi, or gi perfect, are classifiers Clgi correctly classifying all prints of SPgn wrt some gi from $G n$ and Gn perfect are ones correctly 
classifying all prints of SPgn wrt to any gi from $G n$. Other words, all positives of perfect classifiers $C l g i$ are certain utilities wrt to genomic goals gi.

8.2. The assumption of perfect classifiers induce a view on a "paradise" of humans as follows

Ass1. Ideally, if Gn perfect classifiers were available with proper complexity of identification of positives of $+C l g i$, then humans, and assumingly, cellulars too, would do perfectly wrt their genomic goals, thus, would have high degree stability of their being in the stable universe.

Accepting the assumption, we need to answer whether Gn perfect classification can be achieved, while, apparently, we have to preliminarily refine whether do exist gi perfect classifiers for some gi@Gn.

8.3. The existence of gi perfect classifiers for some gi can be argued as follows.

We assume that Sweets, Soars, Hots, etc. are examples of perfect classifiers or their acceptable approximations since some evolutionary utilized realities by a few attributes can be identified uniquely by all humans due to their high genomic equality.

Then, the innate invariance of outputs of sensors of humans to space transformations, for example, positioning, scaling, spinning, or the ability to be activated only by topological invariants, makes it possible to form almost perfect classifiers of some types of realities wrt to Gn.

Seemingly, they are classifiers accumulated in the right hemispheres and are responsible for human and to a variety of extents of cellular innate emotional, communication, survival and other doings.

8.4. Now, let us argue that the entire perfect classification of SPgn is intractable.

Recall, first, that power $\mathrm{n}$ of the units of sensors exceed ... and perfect classification, in general, is enormously hard work since the classifiers have to be identified among the possible $2^{2^{n}}$ ones.

NP completeness of proving the consistency of classifiers wrt certain gi seems analogous to proving that propositional formulas are tautologies

8.4.1. Then, following Kolmogorov, we believe that the units of the universe highly vary in their complexity and include extremely complicated ones.

And human long time attempts to represent the entire universe by comprised, maximum unitary and plain, thus, more manageable classifiers are not successful yet.

At present, those attempts are resulted in revealing only a several perfect classifiers, while mainly ones are represented by incrementally enhancing mosaic of systems of imperfect classifiers, where classifiers of some layers are composed of ad hoc ones of the lower layers by a variety of rels between them.

8.4.2. Each classifier must identify $+C l g i$ in the set of, at least, $2^{n}$ percepts, while their formation is inevitably based on the restricted expertise provided either by the given mpgi matrices or sets of analogously formed classifiers.

Apparently, restricted matrices, as a rule, don't represent target classifiers.

8.4.3. Then, the classifiers either perfect or not, have to be compact enough for the tractable storage and processing time complexities that excludes their storage barely by mpgi matrices even in the case they are correct. In turn, the compactness of original matrices causes loss of their details, thus, inclusion into $+C l g i$ doubtful positives followed by the rise of the mistaken.

Inductive formation, or inference, of classifiers based on the compactors of mpgi matrices, inductors [9], regularly causes imperfectness, since compacting inevitably expands mpgi by not examined positives that with any consequent formation of classifiers rise the risk of incorrectness.

For example, a type of inductors to compact mpgi matrices represent them by equal prepositional formulae Clgi of the types of conjunctive of disjunctive forms composed by logical operators from the attributes. 
Those attributes, in turn, are compressed into possibly min sets preserving yet the distinction between $+C l g i$ and the opposite prints of the initial mpgi.

Due to unexamined expansion, $C l g i$ can identify positives as non-favorable, i.e., no utilities, therefore for $g i$ in formation of consequent classifiers Clgk wrt Clgi, i.e., for $g k=C l g i$, unexamined expanded positives of Clgj will enhance the incorrectness of consequent classifiers. 8.4.4. The above argumentation allows to conclude the following

Ass1. The disclosure of entire perfect classifiers is an intractable problem.

8.5. Note, that intractability of perfect classification is similar to those in combinatorial games, for example in chess, where perfect classifiers of winning, losing and drawing positions can provide perfect chess players while only the number of chess positions in games exceed the number of elementary units of the universe.

8.6. Imperfectness of the models of human classifiers is caused both by the above sources of imperfectness of human classifiers themselves and ones caused by the malicious modeling.

8.6.1. The means of coping with the first type of imperfectness are based on inductive inferring of classifiers by their consisting of regularly enriched matrices representing those classifiers.

Indeed, inductive inferences of classifiers always are based on the restricted mpgi matrices, therefore at each step of applying of inductors, the perfectness of the hypothesis on target classifiers cannot be guaranteed.

Nevertheless, with enriching those matrices with new positives of those classifiers the hypothesis is regularly improved to be consistent with the input matrices and is correctly classifying until failing for some new entry to be modified.

\section{Conclusion}

9.1. Humans become powered enough to question the further types of their being in the universe but they still have no answers whether the solutions are in the corrections of their genomes, discovering new types of human organizations or in transition to a new type of descends, humanoid machines or others.

In parallel, the mystery of cellulars remains unsolved that in the total range from unicells to the highest organisms are predetermined by a type of programs, genomes, and their universal processors.

Acknowledging that genomic reproduction cannot be originated by chance we argue a way of non-cellular origin of realities comparable by cognizing power with humans, which, for the reasons of their stability in the universe, could create a cellular as the alternate to their being.

9.2. Assuming, that the kernel of effective cognition is universal for being in universe, constructive models of that kernel are defined in [2], and certain evidences of their completeness and adequacy are provided, we formulate a hypothesis on the highest constructive cognizers $(h c \operatorname{Cog} s)$ stating that not cellular realities with the highest $h c \operatorname{Cogs}$ can be originated in frame of basics of physicists which, if right, will provide a constructive way to avoid the mystery of origin of cellulars by chance.

We plan to solve $h c \operatorname{Cogs}$ by constructive modeling of the origination of the min classifiers $\operatorname{minCl}$ in early universe and by formation of chains of incrementally complicated mental doings, rooted in minCl and comprising ones of $h \operatorname{Cogs}$.

We interpret mentals introduced in $[1,2,4]$ by the basics of attributive classifying of theoretical computer science.

Referring to some relevant models of classifying we provide an approach to inductive formation of two place relationships and induce guides to formation of mentals, particularly, in the artificial neuron net (ANN) modes. 
We also discuss a the formation/acquisition of systemic classifiers and the ways of coping with imperfect classifying.

9.3.1. Summarizing, we state that the formation of mentals can be illuminated via constructive ways of composing of already available mentals and by means of adapting inductors of certain types [9] to the formation of doins.

Then, we argue that not only 1-place classifiers can be formed inductively, but analogously, at least, 2-place ones, i.e., rels between classified realities with corresponding IDs.

9.3.2. And we also state that, although certain inductors are equally applicable to the formation of 1- and 2-place classifiers of mentals, as well as to ANN, the principles of organization of storages of mentals allowing, particularly, to process net-based inferences are refined only for the mentals.

Thus, the question arises how to organize ANN capable of performing all mental doings available to the mentals and equal to them?

9.3.3. Other words, acknowledging that: mentals are systems over ces doins reducible to the 1-, 2- place relationships (rels) that can be formed inductively, then 1-,2- rels represent the types of classifiers, rules, regularities while their systems represent, particularly, algorithms, abstract classes and abstracts, as well as that mentals are adequate models of thesauruses Th of mental systems (mss) that can be adequately modeled by colored nets netsTh detailed as, say, abstracts or 1-,2- rels, the question raised whether netsTh can be adequately modeled by computers, particularly, be represented by memory of computers in the way that any mental doings processed by netsTh could be equally reproduced in computers.

\section{References}

[1] E. Pogossian, "Towards adequate constructive models of mental systems", 12th International Conference in Computer Science and Information Technologies, Yerevan, pp. 96-101, 2017, as well as IEEE's Xplore electronic library [Online]. Available: https://ieeexplore.ieee.org/document/8312137

[2] E. Pogossian, "Constructing adequate mental models", Transactions of IIAP NAS RA, Mathematical Problems of Computer Science, vol. 50, pp. 35- 51, 2018.

[3] H. Blavatsky, Between Light and Darkness, Жизнь замечательных людей (in Russian). Moscow: Молодаягвардия, 2010.

[4] E. Pogossian, "On the way to dominating cognition", Transactions of IIAP NAS RA, Mathematical Problems of Computer Science, vol. 49, pp. 79-91, 2018.

[5] J. M. R. Parrondo, J. M. Horowitz and T. Sagawa, "Thermodynamics of information", Nature Physics, vol. 11, no. 2, pp. 131-139, 2015.

[6] J. Flavell, The Developmental Psychology of Jean Piaget, D.VanNostrand Comp. Inc., Princeton, N.J., 1962

[7] E. V. Koonin, The Logic of Chance: The Nature and Origin of Biological Evolution, Pearson Education, Inc. Publishing as FT Press Science, 2012.

[8] E. Shrodinger, Mind and Matter, Cambridge, 1956.

[9] E. Pogossian, "Adaptation of combinatorial algorithms", Academy of Sci. of Armenia, p. 293, Yerevan, 1983.

[10] E. Pogossian, "Specifying personalized expertise. international association for development of the information society (IADIS)", International Conference Cognition 
and Exploratory Learning in Digital Age(CELDA 2006), Barcelona, Spain, pp. 151-159, 2006.

[11] H. Marandjian, "A method of synthesis of programs of numeric functions", Mathematical Problems of Cybernetics and Computer, Yerevan, vol. 26, pp. 5-13, 1986.

[12] Discrete Mathematics. Edited by S.Yablonski and O.Lupanov, "Naula", Moscow, 1974.

[13] V.Vapnik and A.Chervonenkis, The Theory of Pattern Recognition, "Naula", Moscow, 1974.

[14] Z. Pylyshyn, Seeing and Visualizing: It's Not What You Think, An Essay on Vision and Visual Imagination, 2004. [Online]. Available: https://ruccs.rutgers.edu/images/personal-zenon-pylyshyn/class-info/bookall.pdf

[15] A. Markov and N. Nagorni, Theory of Algorifms, Nauka, Moscow, 1984.

[16] A. Maltzev, Algorithms and Recursive Functions, Nauka, Moscow, 1965.

[17] E. Pogossian, "Effectiveness enhancing knowledge based strategies for SSRGT class of defense problems", NATO ASI 2011 Prediction and Recognition of Piracy Efforts Using Collaborative Human-Centric Information Systems, Salamanca, Spain, pp. 16, 2011.

[18] S. Grigoryan, N. Hakobyan and H.Vrtanesyan "Object-oriented modeling of systemic classifiers and matching to classifiers", Transactions of IIAP NAS RA, Mathematical Problems of Computer Science, vol. 49, pp.102-107, 2018.

[19] E. Pogossian, M. Hambartsumyan and Y. Harutunyan, A Repository of Units of Chess Vocabulary Ordered by Complexity of their Interpretations. National Academy of Sciences of Armenia, IPIA, research reports, 1974-1980.

Submitted 21.01.2019, accepted 15.04.2019.

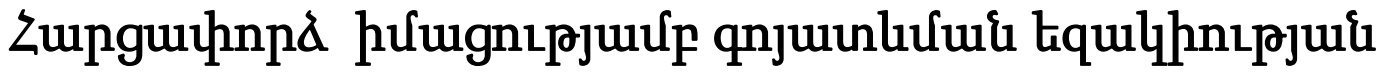 ltipuptipjuil
}

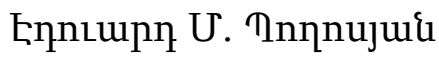

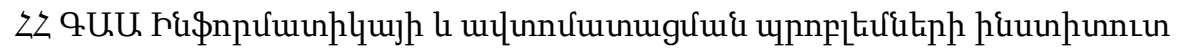 \\ e-mail: epogossi@aua.am
}

\section{Uựhnhnuर}

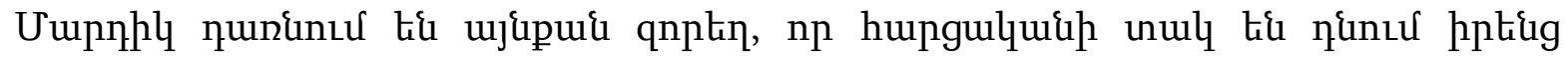

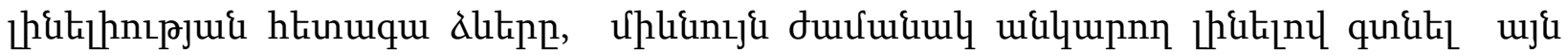

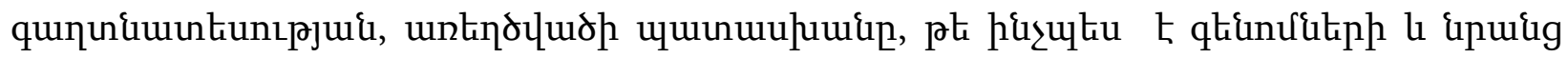




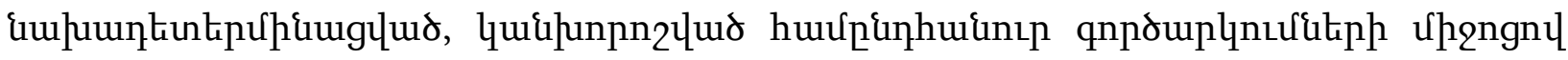

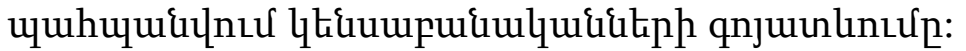

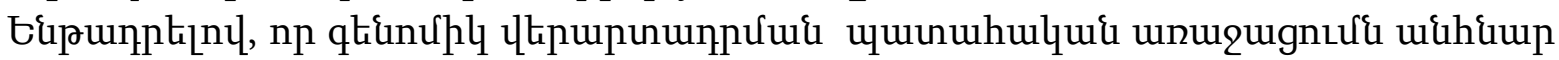

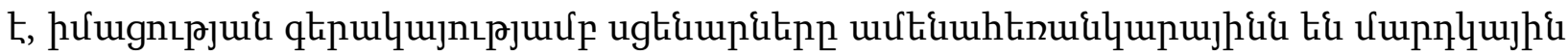

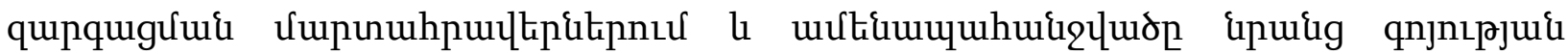

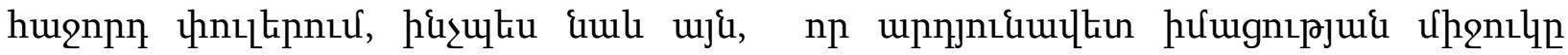

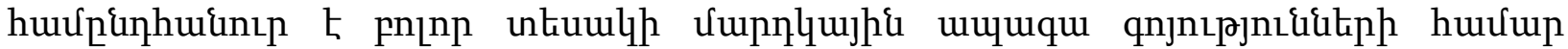

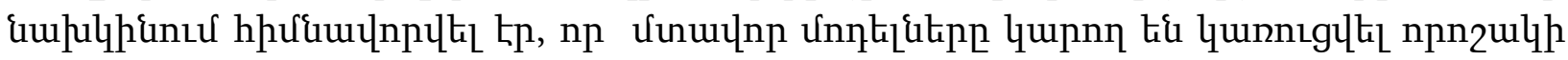
puquujhi nuuulquinqhistiph uhongnı:

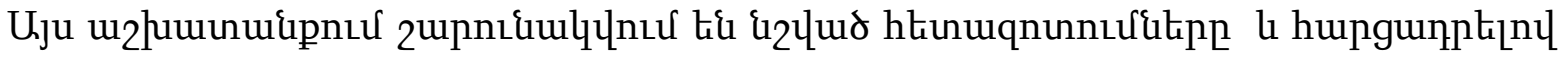

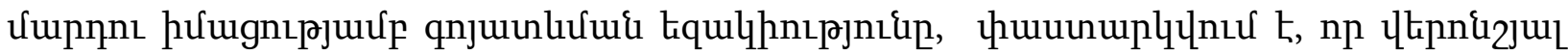

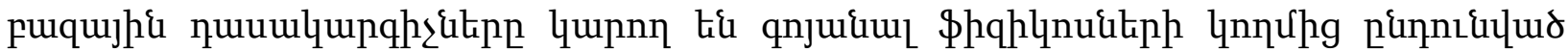

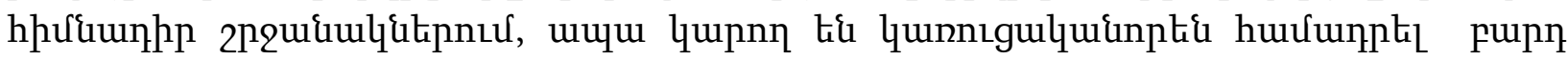
unuulnp hurưuluupqtip:

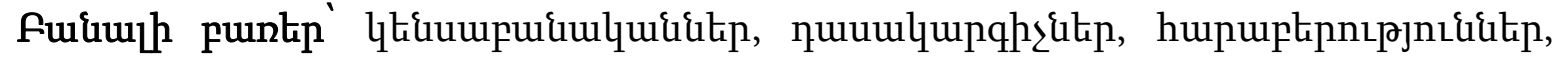

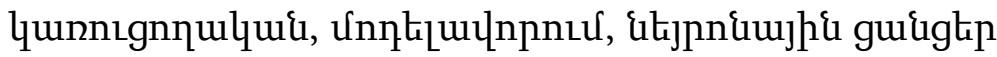

\title{
Уникально ли бытие на основе познания?
}

\author{
Эдвард М. Погосян \\ Институт проблем информатики и автоматизации НАН РА \\ e-mail: epogossi@aua.am
}

\begin{abstract}
Аннотация
Знания человека становятся достаточными, чтобы подвергнуть сомнению устойчивость клеточной организации бытия во вселенной, но не дают ответа, каким образом возникают программы типа генома и их универсального прочтения, позволяющие регулярное воспроизводство клеточных.

Исключая случайность образования геномного воспроизводства и считая, что ядро эффективного познания является универсальным для всех типов негэнтропного бытия, мы сосредотачиваем наши исследования на извлечении и изучении этого ядра.

В данной работе, исходя из ранее обоснованной сводимости ментальных систем к определенным базовым классификаторам, нами допускается возможность поэтапного формирования указанных классификаторов посредством первичных физических реалий и исследуются возможности формирования на их основе более сложных ментальных систем.
\end{abstract}

Ключевые слова: клеточные, классификаторы, отношения, конструктивно, нейронные сети. 